\section{(6) OPEN ACCESS}

\title{
Long-term efficacy and safety of incobotulinumtoxinA injections in patients with cervical dystonia
}

\author{
Dirk Dressler, ${ }^{1}$ Sebastian Paus, ${ }^{2}$ Andrea Seitzinger, ${ }^{3}$ Bernd Gebhardt, ${ }^{3}$ \\ Andreas Kupsch ${ }^{4,5}$
}

\begin{abstract}
${ }^{1}$ Movement Disorders Section, Department of Neurology, Hannover Medical School, Hannover, Germany 2Department of Neurology, University of Bonn, Bonn, Germany

${ }^{3}$ Merz Pharmaceuticals GmbH, Frankfurt am Main, Germany

${ }^{4}$ Clinic and Policlinic for Neurology, Charité-

Universitätsmedizin, Berlin, Germany

${ }^{5}$ Department of Neurology and Stereotactic Neurosurgery, University of Magdeburg, Germany
\end{abstract}

\section{Correspondence to} Professor Dirk Dressler, Head of Movement Disorders Section, Department of Neurology, Hannover Medical School, Hannover 30625, Germany; dressler.dirk@mhhannover.de

Received 4 July 2012 Revised 25 March 2013 Accepted 27 March 2013 Published Online First 18 May 2013

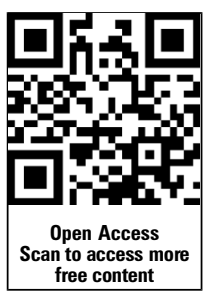

To cite: Dressler $D$, Paus $S$, Seitzinger A, et al. J Neurol Neurosurg Psychiatry 2013;84:1014-1019.

\section{ABSTRACT}

Introduction Previously, controlled trials have demonstrated the efficacy and tolerability of fixed doses of incobotulinumtoxinA (Xeomin, NT 201, botulinum toxin type $A$ free from complexing proteins) to treat cervical dystonia (CD). To explore the clinical relevance of these findings, this study evaluated long-term use of flexible dosing regimens of incobotulinumtoxinA in a setting close to real-life clinical practice.

Methods Patients with $C D$ received five injection sessions of incobotulinumtoxinA using flexible intervals (10-24 weeks) and dosing ( $\leq 300$ Units) based on patients' needs. Outcome measures included Toronto Western Spasmodic Torticollis Rating Scale (TWSTRS), the Dystonia Discomfort Scale (DDS), Investigator Global Assessment of Efficacy (IGAE) and Patient Evaluation of Global Response (PEGR).

Results of 76 patients enrolled (men: $34 \%$; naïve to botulinum toxin: $25 \%$ ), 64 completed the study, receiving treatment over a duration of 49.3-114.1 weeks (total maximum duration: 121 weeks). Mean TWSTRSTotal and DDS scores significantly improved from study baseline to 4 weeks after each injection session (ranges of improvement: TWSTRS-Total: -11.7 to -14.3 ; DDS: -20.2 to -23.0$)$. Up to $81.6 \%$ of investigators rated the efficacy as 'good' or 'very good' (IGAE) and up to $78.9 \%$ of patients rated the treatment response as 'improved' (PEGR). The most common adverse events were dysphagia, nasopharyngitis and headache.

Conclusions In this long-term study, incobotulinumtoxinA was administered using more flexible dosing regimens than those permitted in previous controlled trials. Repeated injections of highly purified incobotulinumtoxinA are effective and well tolerated for the treatment of $C D$ in a setting close to real-life clinical practice.

\section{INTRODUCTION}

Cervical dystonia $(C D)$ is a condition characterised by sustained contractions of the neck and shoulder muscles, and is distinguished from other focal dystonias by a high incidence of associated pain (present in $75 \%$ of patients), which significantly contributes to patient disability. ${ }^{1}$ Intramuscular injections of botulinum toxin (BT) have been shown to be efficacious and well tolerated when used to treat $\mathrm{CD},{ }^{2-7}$ and are therefore recommended as first-line therapy by current treatment guidelines. ${ }^{89}$

BT type A is produced by Clostridium botulinum as a protein complex composed of the $150 \mathrm{kDa}$ neurotoxin and complexing proteins of bacterial origin, including haemagglutinins. ${ }^{10}$
IncobotulinumtoxinA (Xeomin, also known by its Merz drug code NT 201; Merz Pharmaceuticals $\mathrm{GmbH}$, Frankfurt, Germany) is a purified BT type A that differs from other BT drugs in that it contains no complexing proteins, which have been shown to be dispensable for therapeutic efficacy or stability of the neurotoxin. ${ }^{11}{ }^{12}$ Indeed, in head-to-head trials, incobotulinumtoxinA demonstrated comparable efficacy and safety to onabotulinumtoxinA (Botox; Allergan Inc., Irvine, California, USA), in $\mathrm{CD},{ }^{2}{ }^{13}$ blepharospasm ${ }^{13-15}$ and glabellar frown lines ${ }^{16}$ when equivalent doses were used.

As CD is a chronic condition, it is important to document the safety and efficacy of long-term treatment with BT. Recently, a double-blind, randomised trial indicated that repeated injection sessions of incobotulinumtoxinA were efficacious and well tolerated when used to treat $\mathrm{CD}$ over a period of up to 88 weeks. ${ }^{17}{ }^{18}$ However, the total doses used in the trial were fixed (120 mouse units (MU) or $240 \mathrm{MU}$ ) in order to meet the regulatory requirements, and do not represent the approach used in clinical practice, where the dose used is determined based on clinical needs. Therefore, in the present prospective, singlearm study, we sought to further investigate the longterm use of incobotulinumtoxinA in patients with $\mathrm{CD}$ in a setting more representative of real-life clinical practice. Importantly, the study employed flexible dosing regimens that could be adapted according to each patient's individual needs at the discretion of the investigator. Furthermore, the planned study duration was 51-121 weeks: one of the longest evaluation periods for a prospective study of BT type $\mathrm{A}$ in the treatment of $\mathrm{CD}$ to date. We also report on the first long-term use of the Dystonia Discomfort Scale (DDS), a novel patient-rated assessment of the subjective severity of each patient's symptomatology. ${ }^{19}$

\section{METHODS}

This prospective, open-label, single-arm, multicentre Phase IV study was performed between September 2007 and May 2010 at 17 centres in Germany (clinicaltrials.gov identification number NCT00541905). The final study protocol and the informed consent process were approved by the responsible Independent Ethics Committee (The Ethics Commission of the Medical Faculty of Rostock University, Rostock, Germany) and by the competent Regulatory Authority. The study was conducted in accordance with the ethical principles that are outlined in the Declaration of Helsinki, in 
agreement with Good Clinical Practice and the applicable regulatory as well as local legal requirements.

\section{Patients}

Adult patients ( $\leq 75$ years) with $\mathrm{CD}$ of a predominantly rotational form (spasmodic torticollis) and a need for BT injections were eligible. A Toronto Western Spasmodic Torticollis Rating Scale (TWSTRS)-Total score at baseline $\geq 25$ with TWSTRSSeverity score $\geq 10$ and TWSTRS-Disability score $\geq 3$ was also required. Concomitant medications for focal dystonia were permitted if patients had received a stable dose for $\geq 3$ months. Pretreated patients had to have shown a repeated stable response to $\leq 300 \mathrm{MU}$ of onabotulinumtoxinA or incobotulinumtoxinA, or $\leq 1200 \mathrm{MU}$ of abobotulinumtoxinA (Dysport, Ipsen Ltd, Slough, UK); the most recent treatment had to be $\geq 10$ weeks prior to the first injection session of this study.

Patients were excluded if they had previously received BT type B or concomitant BT treatment of any serotype for any other indication. Other exclusion criteria were BT hypersensitivity, neuromuscular disease such as myasthenia gravis, LambertEaton syndrome, amyotrophic lateral sclerosis or severe swallowing disorder of any origin (dysphagia scale score $\geq 3$ ). ${ }^{20}$

\section{Treatment}

During the main period (MP), patients received one injection session with incobotulinumtoxinA, followed by a control visit 4 weeks later. Subsequently, patients entered an extension period (EP), in which they received four additional injection sessions, each followed by a control visit 4 weeks later. Intervals between injection sessions were 10-24 weeks, depending on patients' needs as assessed during telephone interviews. The first telephone interviews were conducted 8 weeks after each injection session. Patients were asked if they deemed a new injection session to be necessary; if so, a visit was scheduled 10 weeks after the last treatment at the earliest. If not, the telephone interviews were repeated fortnightly. The planned total study duration (MP plus EP) was 51-121 weeks.

Patients received individualised, flexible dosing ( $\leq 300 \mathrm{MU}$ total dose, $\leq 50 \mathrm{MU}$ per injection site), determined by the investigator based on physical and neurological examinations. If applicable, the patient's response to any previous BT injections (given before or during this study), including duration of effect and adverse events (AEs), was also taken into consideration. Investigators were free to use electromyographic guidance for target muscle localisation.

\section{Efficacy assessments}

The primary efficacy outcome was the mean improvement in TWSTRS-Total score in the MP (from injection session 1 to the control visit 4 weeks later). The TWSTRS-Total score (range $0-85$ ) is the sum of the severity (range 0-35), disability (range $0-30$ ) and pain (range $0-20$ ) subscores. $^{21}$ Secondary efficacy outcomes included mean changes in TWSTRS-Total score from the study baseline to the control visits in the EP (4 weeks after injection sessions 2-5), as well as mean changes in the TWSTRS-Severity, -Disability and -Pain subscores in the MP and EP. A rater training session was held prior to the study to reduce inter-rater variability, and it was requested that the same investigator performed all assessments for a given patient.

The mean change in DDS score, from baseline to the control visits 4 weeks after each injection session, was also assessed as a secondary efficacy outcome. The DDS documents the subjective severity of each patient's symptomatology in multiples of 5, ranging from 0 (no complaints) to 100 (maximum subjective severity of the untreated condition). ${ }^{19}$ Patients recorded their DDS score every day before going to bed, from the screening visit for the duration of the study. Moreover, the area under the curve (AUC) of DDS score from each injection session to the control visit 4 weeks later was evaluated as a measure of the total burden of a patient's disease.

Additional secondary variables evaluated were Investigator Global Assessment of Efficacy (IGAE) and Patient Evaluation of Global Response (PEGR). The IGAE consists of a 4-point scale ranging from 1 (very good) to 4 (poor), and the PEGR is a descriptive scale ranging from +4 (complete abolishment of signs and symptoms) to -4 (very marked worsening; adapted from Wissel et $a .^{22}$ ) The PEGR response rate was defined as the percentage of patients reporting an improvement of $\geq 2$ (ie, moderate improvement). IGAE and PEGR for each treatment cycle were evaluated at injection visits $2-5$ or the trial termination visit.

\section{Safety assessments}

Patients were asked to report AEs during each visit and telephone interview. In addition, patients were actively asked about swallowing difficulties and evaluated for dysphagia using a 5 -point dysphagia scale adapted from Comella et al, ${ }^{20}$ ranging from 0 (absent, no swallowing difficulties) to 4 (swallowing not possible and resulting in weight loss). Any increase in the dysphagia scale score after baseline was considered to be an AE. Clinical chemistry and haematology tests were conducted at baseline, injection visit 3 and the trial termination visit. Blood samples for the determination of antibodies against BT type A were taken at screening, injection visits $2-5$ and the trial termination visit. Samples taken at screening and at the trial termination visit were tested using a fluorescent immunoassay (FIA). The other samples taken during the study were only tested if the patient had a positive FIA result at the trial termination visit. As the FIA cannot discriminate between neutralising and non-neutralising antibodies, FIA-positive samples were subsequently tested using the mouse hemidiaphragm assay (HDA). ${ }^{23}{ }^{24}$ The Investigator Global Assessment of Tolerability for each treatment cycle was rated on a 4-point Likert scale ranging from 1 (very good) to 4 (poor) at injection visits $2-5$ or the trial termination visit.

\section{Statistical analysis}

Efficacy analyses were carried out based on the full analysis set (FAS; all patients who were enrolled and treated and had a baseline TWSTRS-Total measurement). For mean changes in TWSTRS scores, 95\% CIs based on percentiles of the $t$ distribution were calculated using the last observation carried forward method. Mean changes were regarded as a significant improvement if both ends of the 95\% CI were negative (indicating a reduction in TWSTRS scores). For mean changes in DDS scores, point estimates and parametric 95\% CIs based on the percentiles of the $t$-distribution were calculated; significant improvements in DDS scores were assumed if both ends of the 95\% CI were negative. All changes in TWSTRS and DDS scores are changes from study baseline, that is, before the first incobotulinumtoxinA treatment, to 4 weeks after the respective injection session. Furthermore, correlation analyses between DDS and TWSTRS-Total scores were applied (Bravais Pearson coefficient). IGAE and PEGR were analysed descriptively; differences in PEGR response rates between treatment cycles were analysed by Cochran's Q test. Safety data were based on the safety evaluation set (SES; all patients who were enrolled and treated at least once) and analysed descriptively. 


\section{RESULTS}

\section{Patients}

In total, 82 patients were screened; 76 patients received study medication and were included in the FAS and the SES. Nineteen patients $(25 \%)$ were treatment naïve and 57 patients $(75 \%)$ were pretreated for CD with BT type A. Patient demographics and baseline disease characteristics are shown in table 1 . Twelve patients $(16 \%)$ were prematurely withdrawn from the study due to lack of requirement for further BT injections $(n=2)$, loss to follow-up $(n=2)$, lack of efficacy $(n=1)$, use of BT in another body region $(n=1)$, prior treatment with BT type $B(n=1)$ or other reasons $(n=2)$. Three patients were withdrawn due to the occurrence of AEs: Hodgkin's disease ( $n=1$, not considered to be drug-related), myocardial infarction $(n=1$, not considered to be drug-related) and dysphagia and dyspnoea ( $n=1$, both considered to be mild and drug-related, patient recovered at study end). For the 64 patients (84\%) who completed the study and received all five possible injection sessions, the mean (SD) study duration was 64.3 (11.3) weeks (range: 49.3-114.1 weeks). Mean (SD) total doses ranged from 151.4 (57.8) MU at injection session 1 to 192.2 (69.1) MU at injection session 5. Injection intervals in this study were flexible and based on patients' requests for re-treatment. In deviation from the study protocol, a small number of patients received re-injections less than 10 weeks or more than 24 weeks after their previous treatment. Intervals ranged from 9.9 to 24.0 weeks between injection sessions 1 and 2, 8.1 to 25.1 weeks between sessions 2 and $3,8.4$ to 22.0 weeks between sessions 3 and 4 and 8.1 to 23.6 weeks between sessions 4 and 5 . The mean intervals between injections were stable over the course of the study and ranged from 86.5 (17.7) days between injection sessions 1 and 2 to 89.5 (21.3) days between injection sessions 4 and 5 .

\section{Efficacy}

TWSTRS

IncobotulinumtoxinA injections led to a significant improvement in the mean TWSTRS-Total score between the first injection session and the control visit 4 weeks later of -11.7 (SD $9.8 ; 95 \%$ CI -13.9 to -9.4 ).

Significant improvements were maintained after subsequent injection sessions: mean changes in TWSTRS-Total scores from the study baseline to the respective control visits 4 weeks after injection sessions 2-5 ranged from -13.4 (SD 12.4; 95\% CI -16.3 to -10.6 ) to -14.3 (SD $13.1 ; 95 \%$ CI -17.3 to -11.3 ) (figure 1A). Mean changes in TWSTRS-Severity, TWSTRSDisability and TWSTRS-Pain subscores also indicated consistent significant improvements (figure 1B).

\section{DDS}

Four weeks after each injection session, DDS scores were consistently and significantly improved compared with the study baseline, with mean improvements ranging from -20.2 (SD 27.5; $95 \% \mathrm{CI}-27.1$ to -13.2 ) to -23.0 (SD $26.5 ; 95 \% \mathrm{CI}-29.5$ to -16.5); figure 2). The mean AUC of the DDS scores for the 4 weeks following each injection session was highly stable over the duration of the study, ranging from 1660.1 (SD 725.2; 95\% CI 1479.0 to 1841.3 ) after injection session 4 to 1697.3 (SD $685.9 ; 95 \%$ CI 1538.4 to 1856.2 ) after injection session 1 .

\section{Correlation between TWSTRS total scores and DDS scores}

Correlation analysis of TWSTRS-Total scores and DDS scores over the duration of the study indicated a positive moderate relationship (Bravais Pearson coefficient 0.5482).
Table 1 Baseline demographics and disease characteristics (FAS)

\begin{tabular}{|c|c|}
\hline & $\begin{array}{l}\text { Study population } \\
(\mathrm{n}=76)\end{array}$ \\
\hline Gender: male, $\mathrm{n}(\%)$ & $26(34)$ \\
\hline Mean (SD) age, years & $54.4(10.9)$ \\
\hline Mean (SD) body mass index, $\mathrm{kg} / \mathrm{m}^{2}$ & $25.1(3.8)$ \\
\hline Mean (SD) time since first diagnosis, years & $6.0(6.2)$ \\
\hline Mean (SD) estimated duration of $C D$, years & $9.2(7.2)$ \\
\hline Pretreated with $\mathrm{BT}$ for $\mathrm{CD}, \mathrm{n}(\%)^{*}$ & $57(75)$ \\
\hline Mean (SD) number of injection sessions & $22.4(20.7)$ \\
\hline Mean (SD) time since start of treatment, years & $6.8(5.5)$ \\
\hline \multicolumn{2}{|l|}{ Mean (SD) TWSTRS scores at study baseline } \\
\hline Total & $39.0(10.3)$ \\
\hline Severity & $18.3(3.8)$ \\
\hline Disability & $11.2(5.1)$ \\
\hline Pain & $9.5(4.9)$ \\
\hline Mean (SD) DDS score at study baselinet & $72.2(26.2)$ \\
\hline $\begin{array}{l}\text { Swallowing difficulties at study baseline } \ddagger \text { (dysphagia } \\
\text { scale score }>0 \text { ), } n(\%)\end{array}$ & $7(9)$ \\
\hline \multicolumn{2}{|c|}{$\begin{array}{l}\text { *Formulations received at the last injection session prior to study entry were } \\
\text { incobotulinumtoxinA }(n=32) \text {, abobotulinumtoxinA }(n=14) \text { or onabotulinumtoxinA } \\
(n=11) \text {. } \\
\text { †Baseline DDS scores were available for } 71 \text { patients. } \\
\text { ‡The number of patients with swallowing difficulties at study baseline was based on } \\
\text { the safety evaluation set }(n=76) \text {. } \\
\text { BT, botulinum toxin; CD, cervical dystonia; DDS, Dystonia Discomfort Scale; FAS, full } \\
\text { analysis set; TWSTRS, Toronto Western Spasmodic Torticollis Rating Scale. }\end{array}$} \\
\hline
\end{tabular}

\section{IGAE and PEGR}

The efficacy of incobotulinumtoxinA during each treatment cycle was rated as 'good' or 'very good' by investigators in up to $82 \%$ of cases (table 2). The PEGR response rate for each treatment cycle was up to $79 \%$ (table 2). There were no significant differences in PEGR response rates between treatment cycles $(\mathrm{p}=0.6087)$.

\section{Safety outcomes}

The incidences of the most common AEs (occurring in $\geq 5 \%$ of patients after any injection session) for each treatment cycle are shown in table 3 .

Over all five treatment cycles, the most common drug-related AEs were dysphagia (actively questioned for: $28.9 \%, n=22$ ), neck pain $(10.5 \%, \mathrm{n}=8)$ and muscular weakness $(6.6 \%, \mathrm{n}=5)$.

Dysphagia was also assessed using the dysphagia scale. At study baseline, swallowing difficulties (dysphagia scale score $>0$ ) were reported by seven patients (9\%). An increase in dysphagia scale score between each injection session and the control visit 4 weeks later was noted for seven $(9 \%, n=76)$, five $(7 \%$, $\mathrm{n}=72)$, seven $(10 \%, \mathrm{n}=68)$, three $(5 \%, \mathrm{n}=66)$ and four $(6 \%$, $\mathrm{n}=64)$ patients after injection sessions $1-5$. Swallowing difficulties were mostly mild or moderate (dysphagia scale score of 1 or 2). Two patients who did not report dysphagia at baseline had severe swallowing difficulties that required a change in diet (dysphagia scale score of 3 ). One of these patients reported severe swallowing difficulties 4 weeks after injection session 4 (160 MU incobotulinumtoxinA), and the other patient reported severe swallowing difficulties 8 weeks after receiving $143 \mathrm{MU}$ at injection session 5. For both patients, all occurrences of dysphagia were resolved within 4 weeks.

There were no clinically relevant changes in the clinical laboratory parameter results. At screening, six patients tested positive for BT antibodies in the FIA. Three of these patients also had neutralising antibodies according to the HDA and 

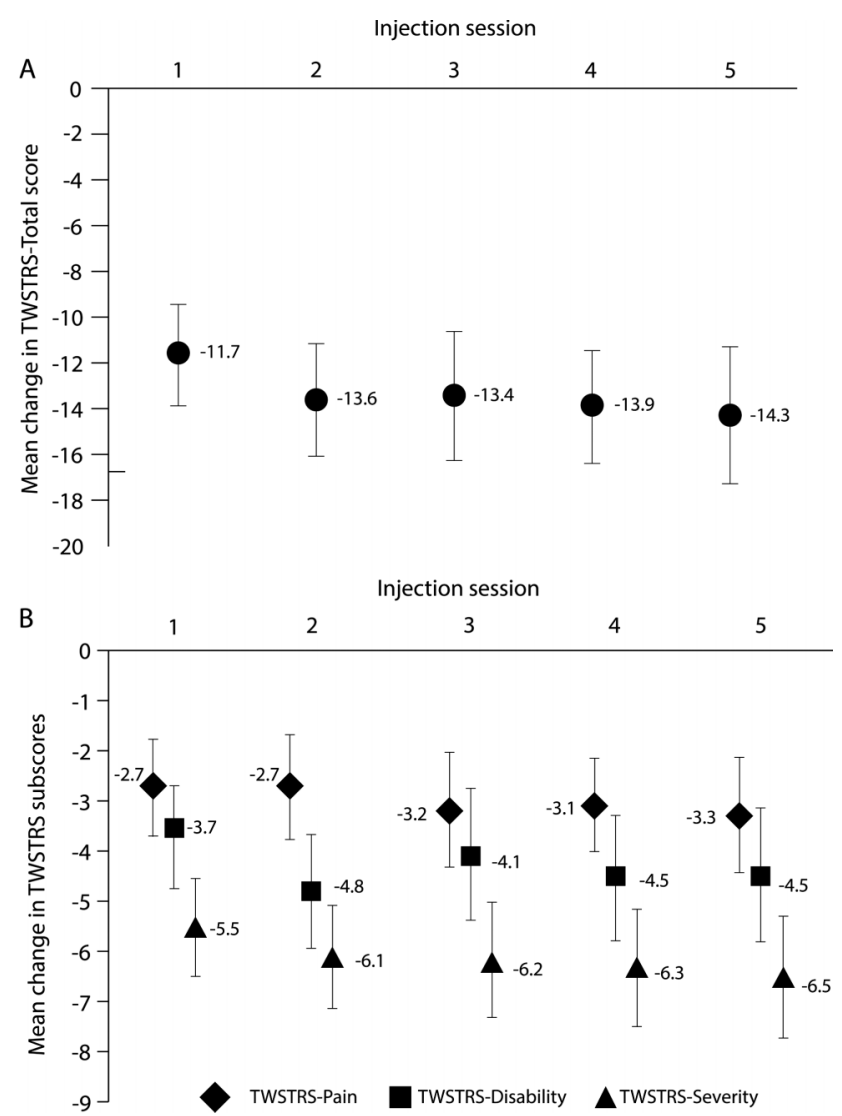

Figure 1 Mean changes in (A) Toronto Western Spasmodic Torticollis Rating Scale (TWSTRS)-Total and (B) TWSTRS-Severity, TWSTRSDisability and TWSTRS-Pain subscores from the study baseline to 4 weeks after each injection session. TWSTRS-Total score ranges from 0 to 85 points and is the sum of the TWSTRS-Severity, TWSTRS-Disability and TWSTRS-Pain subscores. TWSTRS-Severity score ranges from 0 to 35 points, TWSTRS-Disability score from 0 to 30 points and TWSTRSPain score from 0 to 20 points. Error bars indicate the $95 \% \mathrm{Cl}$. TWSTRS, Toronto Western Spasmodic Torticollis Rating Scale.

remained FIA-positive and HDA-positive throughout the study. Of these, two patients consistently experienced improvements of TWSTRS-Total scores between the study baseline and the control visits 4 weeks after each incobotulinumtoxinA treatment. The third HDA-positive patient had an improved

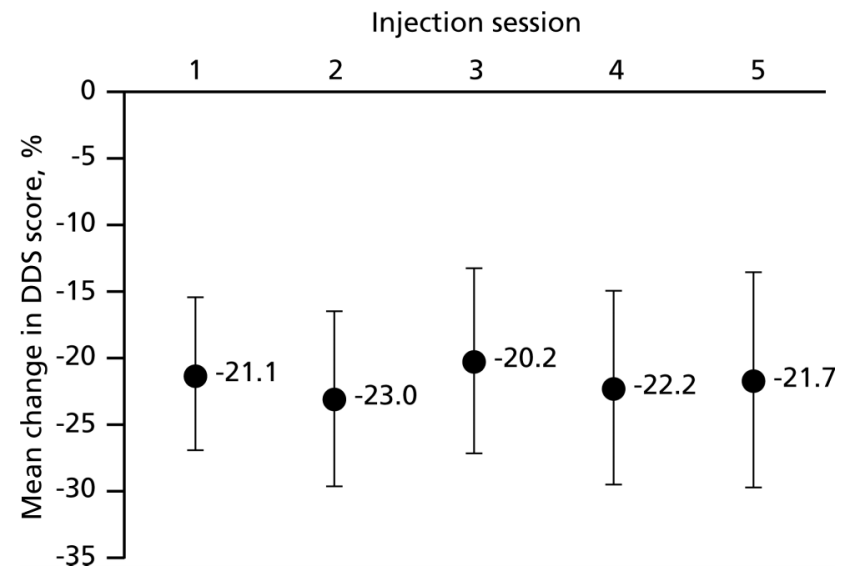

Figure 2 Mean change in Dystonia Discomfort Scale (DDS) scores from the study baseline to 4 weeks after each injection session. DDS scores range from $0 \%$ to $100 \%$. Error bars indicate the $95 \% \mathrm{Cl}$. DDS, Dystonia Discomfort Scale.
Table 2 IGAE and PEGR by treatment cycle (FAS, $n=76$ )

\begin{tabular}{lllllll}
\hline & \multicolumn{7}{l}{ Treatment cycle } \\
\cline { 2 - 6 } & $\mathbf{1}$ & $\mathbf{2}$ & $\mathbf{3}$ & $\mathbf{4}$ & $\mathbf{5}$ \\
\hline IGAE & 68 & 62 & 64 & 67 & 82 \\
$\begin{array}{l}\text { Reports of 'good' or 'very good' efficacy, \% } \\
\text { PEGR }\end{array}$ & 78 & 67 & 72 & 64 & 79 \\
Response rate, \% & & & & & \\
\hline
\end{tabular}

FAS, full analysis set; IGAE, Investigator Global Assessment of Efficacy; PEGR, Patient Evaluation of Global Response.

TWSTRS-Total score 4 weeks after the first but not after subsequent injection visits. The other three patients with FIA-positive results at screening were HDA-negative at screening and throughout the study. Not all FIA-positive samples could be tested in the HDA after the screening. No patients developed new antibodies against BT as assessed using the FIA or neutralising antibodies as per the HDA over the duration of the study.

At injection sessions 2-5 and the trial termination visit, the investigator classified the tolerability of study medication as 'good' or 'very good' for 94\% (68/72), 94\% (64/68), 97\% (64/ $66), 97 \%(62 / 64)$ and $95 \%(70 / 74)$ of patients.

\section{DISCUSSION}

Previously, a controlled clinical trial has demonstrated the safety and efficacy of repeated injections of incobotulinumtoxinA for the treatment of CD when administered at flexible 6-20-week intervals using fixed total doses of 120 or 240 MU. $^{3} 1718$ The dosing in real-life clinical practice is generally more flexible and determined by the physician based on each individual patient's needs. Furthermore, the results of the present prospective, open-label, single-arm, multicentre study confirm the long-term efficacy and tolerability of incobotulinumtoxinA in patients with $\mathrm{CD}$ in a setting that more closely reflects real-life clinical practice. Here, incobotulinumtoxinA provided consistent improvements as assessed on a clinical rating scale for CD (TWSTRS) and other investigator-rated outcomes (IGAE). These correlated with improvements in patient-rated outcomes (PEGR and the DDS), suggesting that patients experience meaningful

Table 3 Incidence of the most common ( $\geq 5 \%$ ) AEs by treatment cycle (SES)

\begin{tabular}{|c|c|c|c|c|c|}
\hline \multirow[b]{2}{*}{$A E, n(\%)$} & \multicolumn{5}{|c|}{ Treatment cycle } \\
\hline & $\begin{array}{l}1 \\
(n=76)\end{array}$ & $\begin{array}{l}2 \\
(n=72)\end{array}$ & $\begin{array}{l}3 \\
(n=68)\end{array}$ & $\begin{array}{l}4 \\
(n=66)\end{array}$ & $\begin{array}{l}5 \\
(n=64)\end{array}$ \\
\hline $\begin{array}{l}\text { Patients with } \geq 1 \\
A E\end{array}$ & $53(70)$ & $50(66)$ & $50(66)$ & $36(47)$ & $35(46)$ \\
\hline Dysphagia* & $14(18)$ & $10(14)$ & $11(16)$ & $7(11)$ & $10(16)$ \\
\hline Nasopharyngitis & $10(13)$ & $11(15)$ & $11(16)$ & $11(17)$ & $8(13)$ \\
\hline Headache & $8(11)$ & $16(22)$ & $9(13)$ & $6(9)$ & $8(13)$ \\
\hline Neck pain & $6(8)$ & $5(7)$ & $6(9)$ & $3(5)$ & $5(8)$ \\
\hline Dry mouth & $4(5)$ & $3(4)$ & $4(6)$ & $1(2)$ & $1(2)$ \\
\hline Back pain & $3(4)$ & $3(4)$ & $4(6)$ & $1(2)$ & $0(0)$ \\
\hline Diarrhoea & $1(1)$ & $0(0)$ & $5(7)$ & $0(0)$ & $2(3)$ \\
\hline
\end{tabular}


symptomatic benefits. It was a limitation of the study design that no placebo control arm was included. However, the overall effectiveness of BT treatment, including incobotulinumtoxinA, versus placebo for $C D$ is well established and treatment effects seen in this study are comparable to other clinical trials. ${ }^{2} 38925$

Moreover, this study encompassed one of the longest evaluation periods (51-121 weeks) to date for a prospective study of $\mathrm{BT}$ in the treatment of $\mathrm{CD}$, and thereby provides an important insight into the outcomes of long-term treatment. While other long-term studies have been reported, these were generally conducted retrospectively ${ }^{26}$ or did not assess clinical efficacy outcomes at the peak effect of treatment. ${ }^{7}$

The present study also supported the long-term usefulness of the DDS- a novel tool to monitor the full-time course of efficacy throughout a BT treatment cycle. The validity and sensitivity of the DDS over the first treatment cycle of this study will be reported elsewhere (Dressler et al 2013, manuscript in preparation). As a self-assessment tool, the DDS can be easily performed by patients on a daily basis without the need to attend a clinic and could potentially provide a valuable and viable method for the evaluation of BT effects on the severity of CD in routine clinical practice.

IncobotulinumtoxinA was generally well tolerated in this study population, with a safety profile similar to that observed in controlled trials of incobotulinumtoxinA in $\mathrm{CD}^{2}{ }^{3} 28$ Importantly, no new or unexpected safety risks emerged with long-term treatment. The most common drug-related AE was dysphagia, a well-known and frequent side effect of BT type A and $\mathrm{B}$ formulations in the treatment of $\mathrm{CD} .^{29-33}$ Moreover, in this study, patients were directly questioned about swallowing difficulties, and additionally, dysphagia was assessed on a rating scale, which may have prompted a greater level of reporting of dysphagia as an AE. The other main drug-related AEs in this study, neck pain and muscular weakness, are also common side effects of BT therapy. ${ }^{29-33}$

Importantly, no accumulation of AEs was observed over the treatment course but the incidence of AEs tended to decrease over time, similar to trends seen in other long-term studies with incobotulinumtoxinA in CD. ${ }^{34}$ This may reflect patients' adjustment to BT treatment over time, an effect that might not be apparent in shorter studies.

Three patients had neutralising antibodies to BT at study baseline according to a mouse HDA; two of these patients remained responsive to incobotulinumtoxinA throughout the study. No patients developed neutralising antibodies during this study, consistent with long-term studies of incobotulinumtoxinA in spasticity $^{35}$ and blepharospasm. Thus, this study supports accumulating evidence that incobotulinumtoxinA, with its low bacterial foreign protein content, is associated with low immunogenicity.

In summary, this study shows that repeated injections of highly purified incobotulinumtoxinA, administered using flexible dosing regimens in a setting close to real-life clinical practice, are effective and well tolerated for the long-term treatment of CD.

Acknowledgements The authors would like to thank all patients and investigators who participated in this study. Editorial support was provided by $\mathrm{Dr}$ Simone Boldt (Complete Medical Communications) and funded by Merz Pharmaceuticals $\mathrm{GmbH}$.

Collaborators Study investigators: Ute Bock, Andres Ceballos-Baumann, Reinhard Dengler, Markus Ebke, Johann Hagenah, Bernhard Haslinger, Martin Hecht, Guenther Heide, Sabine Hellwig, Andreas Kupsch, Sebastian Paus, Johannes Schiefer, Alexander Storch, Peter Vieregge, Thomas Vogt, Jens Volkmann and Hartwig Woldag.
Contributors All authors made substantial contributions to the conception, design and execution of the study, and interpretation of the data. All authors contributed to the intellectual content of the manuscript, reviewed and critically revised it and approved the final submitted version.

Funding The study was funded by Merz Pharmaceuticals $\mathrm{GmbH}$, Frankfurt am Main, Germany.

Competing interests DD received compensation for consultancies from Allergan, Ipsen, Elan/Eisai/Solstice, Merz Pharmaceuticals and Syntaxin. He is a shareholder of Allergan. SP received consultancy fees from Ipsen and Merz Pharmaceuticals. AS is a current employee of Merz Pharmaceuticals. BG is a current employee of Merz Pharmaceuticals. AK received honoraria for lecturing from Allergan, Boehringer Ingelheim, Ipsen, Lundbeck, Medtronic, Merck, Merz Pharmaceuticals, Orion and UCB.

Ethics approval The study was approved by the Ethics Commission of the Medical Faculty of Rostock University, Rostock, Germany.

Provenance and peer review Not commissioned; externally peer reviewed.

Open Access This is an Open Access article distributed in accordance with the Creative Commons Attribution Non Commercial (CC BY-NC 3.0) license, which permits others to distribute, remix, adapt, build upon this work non-commercially, and license their derivative works on different terms, provided the original work is properly cited and the use is non-commercial. See: http://creativecommons.org/ licenses/by-nc/3.0/

\section{REFERENCES}

1 Chan J, Brin MF, Fahn S. Idiopathic cervical dystonia: clinical characteristics. Mov Disord 1991;6:119-26.

2 Benecke $\mathrm{R}$, Jost $\mathrm{WH}$, Kanovsky $\mathrm{P}$, et al. A new botulinum toxin type $\mathrm{A}$ free of complexing proteins for treatment of cervical dystonia. Neurology 2005:64:1949-51.

3 Comella $\mathrm{CL}$, Jankovic J, Truong $\mathrm{DD}$, et al. Efficacy and safety of incobotulinumtoxinA (NT 201, XEOMIN ${ }^{\circ}$, botulinum neurotoxin type $A$, without accessory proteins) in patients with cervical dystonia. J Neurol Sci 2011;308:103-9.

4 Greene P, Kang U, Fahn S, et al. Double-blind, placebo-controlled trial of botulinum toxin injections for the treatment of spasmodic torticollis. Neurology 1990;40:1213-18.

5 Lew MF, Adornato BT, Duane DD, et al. Botulinum toxin type B: a double-blind, placebo-controlled, safety and efficacy study in cervical dystonia. Neurology 1997:49:701-7.

6 Truong D, Duane DD, Jankovic J, et al. Efficacy and safety of botulinum type A toxin (Dysport) in cervical dystonia: results of the first US randomized, double-blind, placebo-controlled study. Mov Disord 2005;20:783-91.

7 Brin MF, Comella CL, Jankovic J, et al. Long-term treatment with botulinum toxin type A in cervical dystonia has low immunogenicity by mouse protection assay. Mov Disord 2008;23:1353-60.

8 Albanese A, Asmus F, Bhatia KP, et al. EFNS guidelines on diagnosis and treatment of primary dystonias. Eur J Neurol 2011;18:5-18.

9 Simpson DM, Blitzer A, Brashear A, et al. Assessment: botulinum neurotoxin for the treatment of movement disorders (an evidence-based review): report of the therapeutics and technology assessment subcommittee of the American Academy of Neurology. Neurology 2008;70:1699-706

10 Inoue K, Fujinaga Y, Watanabe T, et al. Molecular composition of Clostridium botulinum type A progenitor toxins. Infect Immun 1996;64:1589-94.

11 Frevert J. Xeomin is free from complexing proteins. Toxicon 2009;54:697-701.

12 Grein S, Mander GJ, Fink K. Stability of botulinum neurotoxin type A, devoid of complexing proteins. The Botulinum J 2011;2:49-57.

13 Jost WH, Blümel J, Grafe $\mathrm{S}$. Botulinum neurotoxin type A free of complexing proteins (XEOMIN囚) in focal dystonia. Drugs 2007;67:669-83.

14 Roggenkämper $P$, Jost WH, Bihari $K$, et al. Efficacy and safety of a new Botulinum Toxin Type A free of complexing proteins in the treatment of blepharospasm. J Neural Transm 2006;113:303-12.

15 Wabbels B, Reichel G, Fulford-Smith A, et al. Double-blind, randomised, paralle group pilot study comparing two botulinum toxin type $A$ products for the treatment of blepharospasm. J Neural Transm 2011;118:233-9.

16 Sattler G, Callander MJ, Grablowitz D, et al. Noninferiority of incobotulinumtoxinA, free from complexing proteins, compared with another botulinum toxin type $A$ in the treatment of glabellar frown lines. Dermatol Surg 2010;36(Suppl 4):2146-54.

17 Evidente VG, Truong $D$, Fernandez $\mathrm{H}$, et al. Efficacy and safety of repeated incobotulinumtoxinA injections in subjects with cervical dystonia previously treated with botulinum toxin [abstract]. Neurology 2011;76(Suppl 4):PA540.

18 Fernandez HH, Comella C, Brodsky M, et al. Efficacy and safety of repeated incobotulinumtoxinA injections in subjects with cervical dystonia previously naive to botulinum toxin treatment [abstract]. Neurology 2011;76(Suppl 4):PA330.

19 Interdisziplinärer Arbeitskreis Bewegungsstörungen. The Dystonia Discomfort Scale (DDS). http://dds.iabnetz.de (accessed 9 Jan 2012). 
20 Comella CL, Tanner CM, DeFoor-Hill L, et al. Dysphagia after botulinum toxin injections for spasmodic torticollis: clinical and radiologic findings. Neurology 1992:42:1307-10.

21 Consky ES, Lang AE. Clinical assessments of patients with cervical dystonia. In: Jankovic J, Hallett M, eds. Therapy with botulinum toxin. New York, NY: Marcel Dekker, Inc., 1994:211-37.

22 Wissel J, Müller J, Dressnandt J, et al. Management of spasticity associated pain with botulinum toxin A. J Pain Symptom Manage 2000;20:44-9.

23 Dressler D, Dirnberger G. Botulinum toxin antibody testing: comparison between the immunoprecipitation assay and the mouse diaphragm assay. Eur Neurol 2001:45:257-60

24 Sesardic $D$, Jones RG, Leung $T$, et al. Detection of antibodies against botulinum toxins. Mov Disord 2004;19(Suppl 8):S85-91.

25 Lew MF, Chinnapongse R, Zhang $Y$, et al. RimabotulinumtoxinB effects on pain associated with cervical dystonia: results of placebo and comparator-controlled studies. Int J Neurosci 2010;120:298-300.

26 Jankovic J, Schwartz K. Botulinum toxin injections for cervical dystonia. Neurology 1990:40:277-80

27 Mohammadi B, Buhr N, Bigalke $H$, et al. A long-term follow-up of botulinum toxin A in cervical dystonia. Neurol Res 2009;31:463-6.
28 Truong D, Fernandez HH, Grafe SK. IncobotulinumtoxinA (NT-201) injections are safe and effective in adult subjects with cervical dystonia across dosing intervals in a repeated-dose study [abstract]. Mov Disord 2011;26(Suppl 2):PS211.

29 Allergan, Inc. Botox® US Prescribing Information. http://www.accessdata.fda.gov/ drugsatfda docs/label/2011/103000s5232lbl.pdf (accessed 18 Jun 2012).

30 Ipsen Biopharm, Ltd. Dysport US Prescribing Information. http://www.accessdata.fda. gov/drugsatfda_docs/label/2012/125274s0052lbl.pdf (accessed 19 Jun 2012).

31 Merz Pharmaceuticals GmbH. Xeomin® US Prescribing Information. http://www. accessdata.fda.gov/drugsatfda_docs/label/2011/125360s007/bl.pdf (accessed 19 Jun 2012).

32 Solstice Neurosciences, Inc. Myobloc US Prescribing Information. 2009

33 Dressler D. Botulinum toxin for treatment of dystonia. Eur J Neurol 2010;17 (Suppl 1):88-96.

34 Grafe S, Hanschmann A. Safety and efficacy of repeated NT 201 (Botulinum neurotoxin type $A$ free from complexing proteins) injections of patients with cervical dystonia: a first long-term safety analysis [abstract]. Neurology 2010;74:PA88.

35 Kañovský P, Slawek J, Denes Z, et al. Efficacy and safety of treatment with incobotulinum toxin $A$ (botulinum neurotoxin type $A$ free from complexing proteins; NT 201) in post-stroke upper limb spasticity. J Rehabil Med 2011;43:486-92. 